\title{
Adoption of Diffusion Tensor Imaging under Optimized Fuzzy C-Means Cluster Algorithm in Intracerebral Benign and Malignant Tumor Resection
}

\author{
Yasong Liu $\mathbb{i D}^{1}$ and Wentao $\mathrm{Xu} \mathbb{i}^{2}$ \\ ${ }^{1}$ Department of Neurosurgery, The First People's Hospital of Ningyang County, Ningyang 271400, Shandong, China \\ ${ }^{2}$ Department of Neurosurgery, Heze Hospital of Traditional Chinese Medicine, Heze 274000, Shandong, China \\ Correspondence should be addressed to Wentao Xu; 132020430346@st.sdju.edu.cn
}

Received 8 June 2021; Revised 30 June 2021; Accepted 28 July 2021; Published 2 August 2021

Academic Editor: Gustavo Ramirez

Copyright ( 2021 Yasong Liu and Wentao Xu. This is an open access article distributed under the Creative Commons Attribution License, which permits unrestricted use, distribution, and reproduction in any medium, provided the original work is properly cited.

\begin{abstract}
The study aimed to analyze the application of diffusion tensor imaging (DTI) in the surgery of benign and malignant intracranial tumors through improved fuzzy C-means (FCM). First, a method of combining the maximum and minimum distances was proposed to improve the FCM algorithm. Then, the optimized FCM was applied to the diffusion tensor imaging (DTI) diagnosis. The patients were rolled into the benign tumor group and the malignant tumor group, and relevant parameters were compared. Finally, the postoperative total resection rate and disability rate of the DTI experimental group and the traditional control (Ctrl) group were evaluated. It was found that the segmentation accuracy of the optimized FCM algorithm was higher than traditional one and the obtained corpus callosum edge contour was clearer. In 63 patients with intracranial space, there were obvious differences in pairwise comparison of meningioma and glioma, metastatic tumor's apparent diffusion coefficient (ADC) value, relative apparent diffusion coefficient ( $\mathrm{r}$ ADC) value, and relative anisotropy fraction ( $\mathrm{r}$ FA) $(P<0.05)$. In terms of the ADC, $r$ ADC, and $r$ FA values of tumor parenchymal area, those of benign tumors were larger than malignant tumors $(P<0.05)$. The ADC value $(8.21 \pm 1.87)$ and $r$ FA value $(1.36 \pm 0.41)$ of the contralateral normal white matter area of malignant tumor were greater than the ADC value $(7.23 \pm 2.31)$ and $\mathrm{r}$ FA value $(0.61 \pm 0.24)$ of the peritumor white matter area, with statistically significant differences $(P<0.05)$. The total cut rates of the experimental group and the Ctrl were $87.5 \%$ and $54.84 \%$, and the disability rates were $6.25 \%$ and $34.38 \%$. In conclusion, the optimized FCM has high accuracy. The ADC, $r$ ADC, and r FA values of DTI are important in the diagnosis of intracranial tumors. Besides, DTI can improve the survival rate in guiding intracranial tumor resection.
\end{abstract}

\section{Introduction}

The cranium is an important regulating center and an important organ of human consciousness and thinking. Because the intracranial space is very narrow, all spaceoccupying lesions will invade and destroy the surrounding normal brain tissue. As a result, it will cause some symptoms such as increased intracranial pressure and focal localization signs [1]. Intracranial tumors can be divided into two types: primary intracranial tumors and secondary intracranial tumors. The former refers to tumors that occur in intracranial tissues, while the latter refers to certain tumors that extend or metastasize from the body to the brain [2]. Glioma, meningioma, and metastatic tumor are all common intracranial tumors. Due to the difference in tumor morphology, size, and lesion location, different tumors have different treatment methods $[3,4]$. Therefore, preoperative precise qualitative is important in the selection of tumor treatment methods, guiding the formulation of treatment plans and evaluating the prognosis of patients.

Magnetic resonance imaging (MRI) has the advantages of high soft tissue resolution, arbitrary layer imaging, no radiation damage, and multiparameter contrast mechanism [5]. However, clinically, MRI is difficult to diagnose when 
the diseases are different, which requires a new MRI technology to assess the extent of tumor invasion $[6,7]$. DTI is a new imaging technology that uses the principle of water molecular diffusion to detect the microstructure of living tissues on the basis of diffuse weighted imaging (DWI). It features being noninvasive and no need for contrast agents $[8,9]$. FCM is more used in image segmentation, and more information of the required area can be retained during the segmentation process. Ali et al. (2015) [10] used morphological pyramid to combine multi-resolution image and original image, and segmented human brain image by the FCM algorithm. Then, the accuracy of the final result was improved. The traditional FCM algorithm has randomness in the selection method of the initial center point. The initial center point can be determined by the combination of the maximum and minimum distances. The FCM algorithm is optimized to improve the accuracy of the tissue region segmentation in the DTI data, which provides excellent analytical methods [11, 12]. Chuang et al. (2006) [13] added the spatial information function and, at the same time, used the fuzzy C-means algorithm to make the segmented area more uniform and reduce the interference noise points.

In this study, the traditional FCM clustering algorithm was optimized by combining the maximum and minimum distances, so as to determine the initial center point. Then, the optimized FCM clustering algorithm was applied to DTI images of benign and malignant intracranial tumors.

\section{Materials and Methods}

2.1. Selection of Samples. In this study, patients with intracranial occupancy from January 2018 to January 2020 in the hospital were selected as the research subjects. All patients had not received chemotherapy, radiotherapy, and other anti-tumor treatments before surgery. There were 63 patients (27 males and 36 females) with intracranial spaceoccupying patients, with an average age of $55.37 \pm 9.83$ years old. The 63 patients were rolled into an experimental group of 32 cases (14 males and 18 females) and a Ctrl of 31 cases (13 males and 18 females). The average age of the experimental group was $49.57 \pm 7.36$ years, and that of the Ctrl was $52.36 \pm 12.69$ years old. Patients in the experimental group underwent conventional MRI scans, enhanced scans, and DTI scans before the operation. After the imaging examination, the optimized FCM clustering algorithm and the combination of neuron avigation system and MRI information were used for tumor resection. The Ctrl accepted conventional MRI scans and enhanced scans. Besides, traditional methods were adopted for tumor resection. The study had got permission from the Medical Ethics Committee of hospital, and all patients were informed of their condition and signed informed consent forms.

Inclusion criteria are as follows: (i) the patient was diagnosed as intracranial occupancy through surgery or histopathology; (ii) the data of conventional MRI scan, enhanced scan, and DTI examination were complete; (iii) patients who accepted follow-up; (iv) patients without contraindications to MRI scanning.
Exclusion criteria are as follows: (i) liver and kidney dysfunction; (ii) there were contraindications to magnetic resonance scanning; (iii) patients who had been treated before surgery; (vi) patients who had obvious mental disorders.

2.2. MRI Scanning Method and Parameters. Before the examination, the basic information of the patient was checked and whether the patient had undergone surgery was asked, and the patient was explained the conditions that may occur during the examination.

All patients lied in a supine position in the multichannelphased array coil of the head. The two upper limbs were placed next to the body. The patients were instructed to keep the brakes during the scanning process.

Patients in the experimental group underwent conventional MRI scans and DTI scans. After Gd-DTPA (Germany) was intravenously injected into the middle of the elbow using a special syringe for MRI, the scans in sagittal, axial, and coronal positions were performed. The tumor was removed during the operation with the help of neuron avigation technology. The patients in Ctrl had conventional MRI scans and enhanced scans. The tumors were removed using traditional methods during the operation.

DTI scan parameters are as follows: the repetition time (TR) was $4800 \mathrm{~ms}$, the matrix was $570 \times 570$, the field of view was $250 \mathrm{~cm} \times 250 \mathrm{~cm}$, and the scan time was $369 \mathrm{~s}$.

2.3. FCM Clustering Algorithm. The whole lesion was a classic area selection method in imaging research. The accuracy of segmentation was related to the accuracy of subsequent doctors' diagnosis. The FCM clustering algorithm can retain more information about specific areas during the segmentation process, which is important for precise segmentation. The expression of FCM is as follows:

$$
J \tau(U, P)=\sum_{i=1}^{S} \sum_{k=1}^{R}(u k i)^{\tau}\left\|x_{i}-p_{k}\right\|^{2},
$$

where $U$ represents the set of membership degrees, $P$ represents the set of cluster center points, $P=\left\{p_{1}, p_{2}, p_{3}, \ldots, p_{R}\right\}$, $u_{k i}$ indicates that a sample point is a membership function of a certain type, $\tau$ indicates a fuzzy weighted index, 2 is the default value, and $\left\|x_{i}-p_{k}\right\|^{2}$ indicates the clustering distance from the $i$-th data point to the $k$-th center point, which is measured by the Euclidean measurement method. To find the extreme value of (1), since the sum is 1 of the membership degrees from a certain sample to all classes, the Lagrangian multiplier method is adopted to obtain the equation below:

$$
1-\sum_{k=1}^{R} u_{k i}=0
$$

Substituting (2) into (1) can obtain the below equation: 


$$
J \tau(U, P)=\lambda\left(1-\sum_{k=1}^{R} u_{k i}\right)+\sum_{i=1}^{S} \sum_{k=1}^{R}(u k i)^{\tau}\left\|x_{i}-p_{k}\right\|^{2} .
$$

The partial derivation of $\lambda$ in (3), the degree of membership, and the center point function can obtain the equation below:

$$
\begin{aligned}
& \frac{\partial J}{\partial \lambda}=1-\sum_{k=1}^{R} u_{k i}, \\
& \frac{\partial J}{\partial u_{k i}}=-\lambda+\tau u_{k i}^{\tau-1}\left\|x_{i}-p_{k}\right\|^{2}, \\
& \frac{\partial J}{\partial u_{k i}}=2 \sum_{k=1}^{R}\left(u_{k i}\right)^{\tau}\left(p_{k}-x_{i}\right) .
\end{aligned}
$$

If the above three partial derivative equations are $e$ equal to 0 , the equation below is obtained by (5):

$$
u_{k i}=\left(\frac{\lambda \times(1 / \tau)}{\left\|x_{i}-p_{k}\right\|^{2}}\right)^{-(\tau-1)} \text {. }
$$

From (7), the equation below is obtained:

$$
p_{k}=\frac{\sum_{i=1}^{S} u_{k i}^{\tau} \times x i}{\sum_{i=1}^{S} u_{k i}^{\tau}},
$$

where $P_{k}$ represents the cluster center point function.

When (7) is substituted into (4), the equation below is obtained:

$$
1-\sum_{k=1}^{R}\left(\frac{\lambda \times(1 / \tau)}{\left\|x_{i}-p_{k}\right\|^{2}}\right)^{-(\tau-1)}=0 .
$$

Then, the equation below is obtained by simplifying (9):

$$
\left(\lambda \times \frac{1}{\tau}\right)^{-(\tau-1)}=\frac{1}{\sum_{j=1}^{R}\left(1 /\left\|x_{i}-p_{k}\right\|^{-2(\tau-1)}\right)} .
$$

When (10) is substituted into (7), the equation below is obtained:

$$
u_{k i}=\frac{1}{\sum_{j=1}^{R}\left(\left\|x_{i}-p_{k}\right\| /\left\|x_{i}-p_{j}\right\|\right)^{-2(\tau-1)}} .
$$

Therefore, the membership function can be finally obtained as $u_{k i}$ in (11).

\subsection{Intracranial Tumor Tissue Segmentation Algorithm.} The traditional FCM has randomness in the selection method of the initial center point. The initial cluster center point can be determined by combining the maximum and minimum distances to optimize the FCM algorithm. The specific method steps are as follows: (i) For $u$ data points, $l u$ is the set of all data points, expressed as $l u=\{x 1, x 2, x 3, \ldots$, $x u\}$. One of the $u$ data points is selected as the center point of the first type, denoted as $N\{1\}$, where $N$ is the set of center points. (ii) The Euclidean distance is calculated from the remaining data points to the $N\{1\}$ th, so that the second cluster center point is the data point corresponding to the maximum distance, denoted as $N\{2\}$. The Euclidean distance is denoted by D. (iii) The Euclidean distances are calculated from all remaining data points $x_{i}$ in the data set $l_{u}$ to the cluster center points $N\{1\}$ and $N\{2\}$. They are denoted as $d_{i 1}$ and $d_{i 2}$. Then, the minimum of $d i_{1}$ and $d i_{2}$ is put into set $B$, which is expressed as $B=\left\{\min \left(d i_{1}, d i_{2}\right)\right\}$. Then, $x_{i}$ corresponding to the maximum value from set $B$ is calculated. It is used as the third-type center point, expressed as $N\{3\}$.

2.5. Image Processing and Data Analysis. The DTI image was processed by function tool software to obtain FA and ADC figures. Then, the FA and ADC of the contralateral normal white matter area, tumor parenchymal area, and tumor narration area were measured. After that, the corresponding r FA and $r$ ADC were obtained by calculation.

The function tool software was used to process the DTI image. Then, the area where the tumor was located was selected to be the area of interest. The countermeasure white matter area of interest area was found through symmetry. The fiber bundle was established by clicking fiber tracking. At the same time, the color image was selected on the DTI image. Then, the shape of intracranial fiber bundles was observed.

2.6. Statistical Methods. The data were processed by SPSS 19.0 , and mean \pm standard deviation (SD) was how the measurement data were expressed. The differences were analyzed by $t$-test between the indicators of benign tumors and malignant tumors, and between the normal white matter areas and peritumoral white matter areas on the contralateral side of the tumor. Comparison of tumor resection rate and postoperative disability rate was realized by the $\chi^{2}$ test. When $P<0.05$, the difference is statistically significant.

\section{Results}

3.1. The Segmentation Results. The original MRI of the brain tumor, the image segmented by the traditional FCM clustering algorithm, and the image segmented by the optimized FCM clustering algorithm were compared. Figure $1(a)$ is the original magnetic resonance image, and Figure 1(b) is the segmentation result of traditional FCM clustering algorithm. Figure $1(\mathrm{c})$ is the result of the optimized FCM clustering algorithm segmentation. It was evident from DTI image segmentation that the edges of the corpus callosum in the images segmented by the traditional FCM clustering algorithm were fuzzy and rough. There were many areas with segmentation errors. However, the edges were clear and smooth of the corpus callosum obtained by the optimized FCM clustering algorithm segmentation. There were few incorrectly segmented regions. It can be inferred that the optimized FCM algorithm can improve the accuracy of segmentation. 


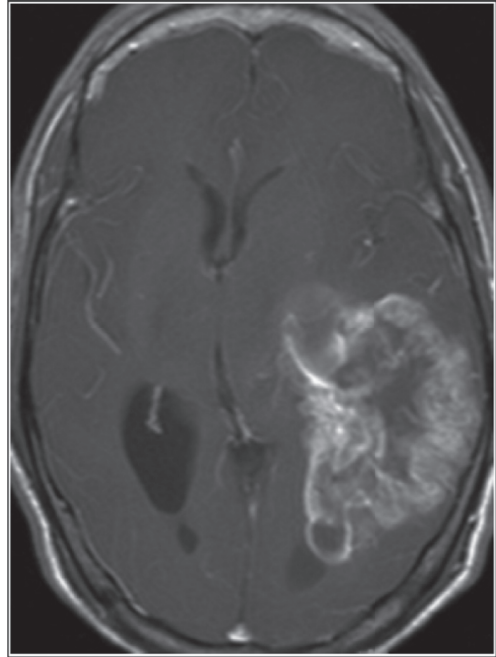

(a)

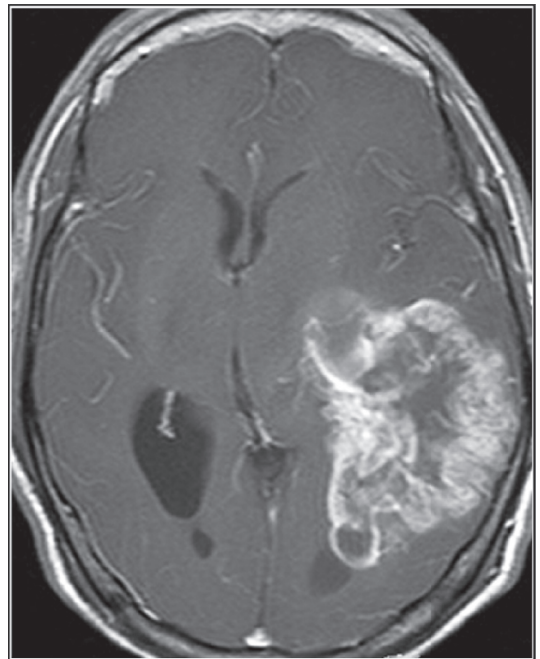

(b)

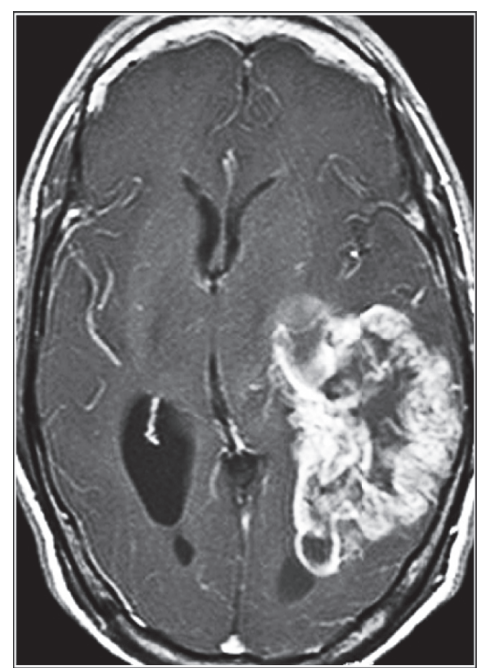

(c)

FIGURE 1: MRI images of intracranial tumors. (a) is the original MRI images; (b) is the result of traditional FCM clustering algorithm segmentation; (c) is the result of optimized one. Note: it is a magnetic resonance image of an intracranial tumor in a 47-year-old woman.

3.2. Comparison of FA, ADC, $r F A$, and $r$ ADC Values in Parenchymal Area. The types of tumors were as follows: glioma (31 cases), meningiomas (19 cases), and metastases (13 cases). Meningiomas are classified as benign brain tumors, and metastatic tumors and gliomas are classified as malignant brain tumors. The data revealed that the $\mathrm{ADC}$ value of glioma were $3.97 \pm 1.83, \mathrm{r}$ ADC value was $1.23 \pm 0.52$, FA value was $0.17 \pm 0.03$, and $\mathrm{r}$ FA value was $0.62 \pm 0.29$. The ADC value of meningioma was $7.53 \pm 1.21, \mathrm{r}$ ADC value was $2.01 \pm 0.93$, FA value was $0.31 \pm 0.23$, and $r$ $\mathrm{FA}$ value was $1.36 \pm 0.77$. The ADC value of metastatic tumor was $5.63 \pm 3.13$, r ADC value was $1.81 \pm 1.22$, FA value was $0.12 \pm 0.09$, and $r$ FA value was $0.87 \pm 0.39$. As shown in Figure 2, the ADC value of meningioma was higher than that of glioma $(P<0.05)$; and the pairwise comparison between $r$ $\mathrm{ADC}$ value, $\mathrm{r}$ FA value of meningioma and $\mathrm{r}$ ADC value and r FA values of glioma showed obvious differences $(P<0.05)$. In Figure 3, the ADC value of meningiomas was higher than that of metastatic tumors $(P<0.05)$. The pairwise comparison between the $\mathrm{r}$ ADC value and $\mathrm{r}$ FA value of meningioma and the $\mathrm{r}$ ADC value and $\mathrm{r}$ FA value of metastatic tumors showed obvious differences $(P<0.05)$. However, there was no obvious difference in FA values of meningioma, glioma, and metastatic tumors $(P>0.05)$.

3.3. Comparison of FA Value, ADC Value, $r$ FA Value, and $r$ ADC Value of Parenchymal Area in Patients with Benign and Malignant Tumors. The meningiomas were classified as benign brain tumors, and metastases and gliomas as malignant brain tumors. The differences between the various indicators in the parenchymal area of benign and malignant tumors were compared. It can be inferred that the ADC value of benign tumors was $7.53 \pm 1.21, \mathrm{r}$ ADC value was $2.01 \pm 0.93$, FA value was $0.31 \pm 0.23$, and $r$ FA value was $1.36 \pm 0.77$; the ADC value of malignant tumor was $74.87 \pm 1.12$, r ADC value was $1.54 \pm 0.76$, FA value was

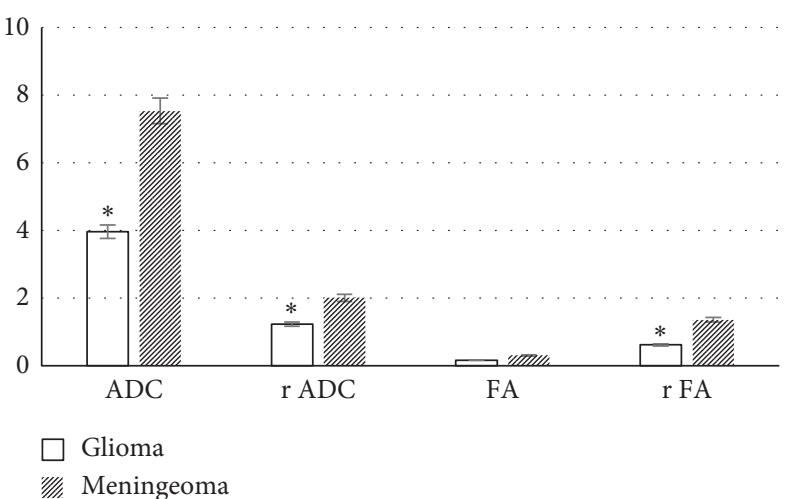

Figure 2: Comparison of FA, ADC, r FA, and $r$ ADC values between meningioma and glioma parenchymal areas. Note: ${ }^{*}$ meant that the difference was obvious in contrast with the meningioma group $(P<0.05)$ (with the same meaning in Figure 4).

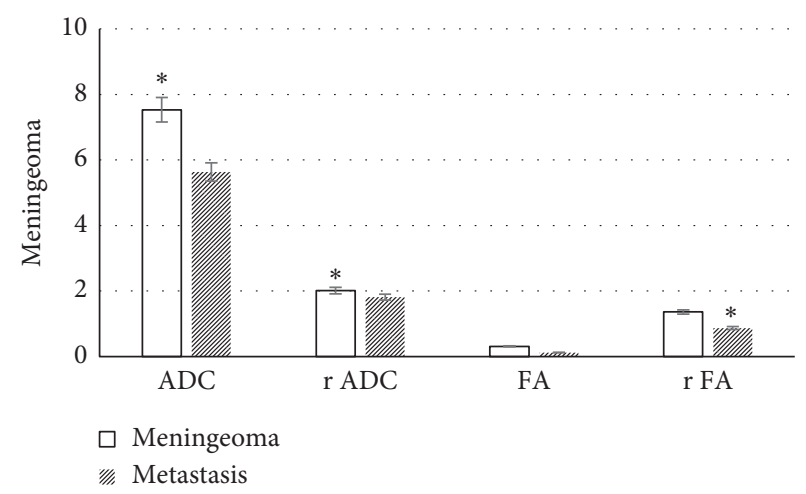

Figure 3: Comparison of FA value, ADC value, $r$ FA value, and $r$ $\mathrm{ADC}$ value in parenchymal area of meningioma and metastasis.

$0.15 \pm 0.05$, and $\mathrm{r} F A$ value was $0.75 \pm 0.31$. As shown in Figure 4, the ADC value, $\mathrm{r}$ FA value, and $\mathrm{r}$ ADC value of benign tumors were obviously higher than those of 


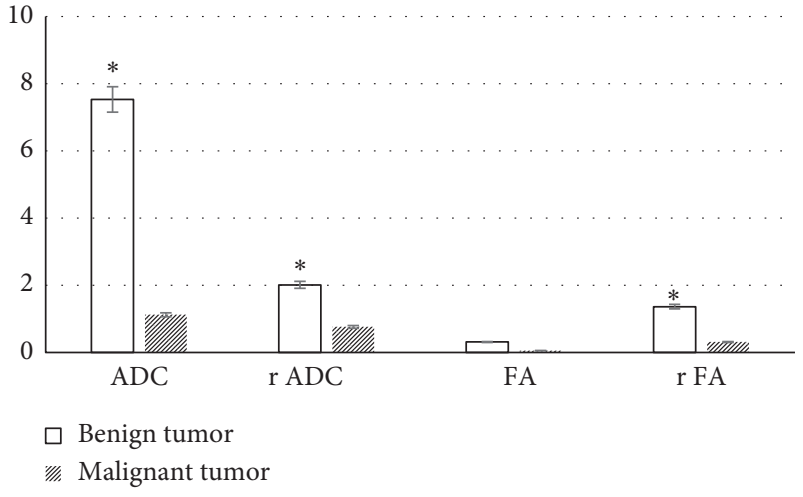

Figure 4: Comparison of FA, ADC, $r$ FA, and $r$ ADC values in parenchymal areas of patients with benign and malignant tumors. Note: ${ }^{*}$ indicated that the difference was obvious compared to the malignant tumor group $(P<0.05)$.

malignant tumors $(P<0.05)$. However, the FA value of benign tumors was not obviously different from that of malignant tumors $(P<0.05)$.

3.4. Comparison of Various Indexes of Normal White Matter Area and Peritumoral White Matter Area on the Contralateral Side of Malignant Tumor. The FA value, ADC value, $r$ FA value, and $\mathrm{r}$ ADC value of the contralateral normal white matter area and peritumoral white matter area of the intracranial tumor were measured and calculated. It can be inferred that the ADC value of the contralateral normal white matter area was $8.21 \pm 1.87, \mathrm{r}$ ADC value was $1.63 \pm 0.35$, FA value was $0.53 \pm 0.21$, and $\mathrm{r} F A$ value was $1.36 \pm 0.41$; the $\mathrm{ADC}$ value of peritumoral white matter area was $7.23 \pm 2.31, \mathrm{r}$ ADC value was $8.27 \pm 1.03$, FA value was $0.34 \pm 0.11$, and $\mathrm{r} F A$ value was $0.61 \pm 0.24$. As shown in Figure 5, the ADC value and $\mathrm{r}$ FA value of the contralateral normal white matter area were higher than the those of the peritumoral white matter area $(P<0.05)$; the $\mathrm{ADC}$ value of the contralateral normal white matter area was significantly lower than that of the peritumoral white matter area $(P<0.05)$; while there was no obvious difference in the FA values between the two areas $(P>0.05)$.

3.5. Comparison of Postoperative Resection Rates. 63 patients were rolled into an experimental group of 32 cases (14 males and 18 females) and a Ctrl of 31 cases (13 males and 18 females). Patients in the experimental group received conventional MRI scans, enhanced scans, and DTI scan before surgery. After imaging examination, the optimized FCM clustering algorithm and the combination of neuron avigation system and magnetic resonance information technology were used for tumor resection. The Ctrl accepted conventional magnetic resonance plain scan and enhanced scan. The traditional methods were adopted for tumor resection. The tumor resection rates were as follows: in the experimental group, 28 cases were completely resected, 4 cases were partially resected, and the total resection rate was 87.5\%. In Ctrl, 17 cases were completely resected, and 14 cases partially resected. The resection rate was $54.84 \%$. As

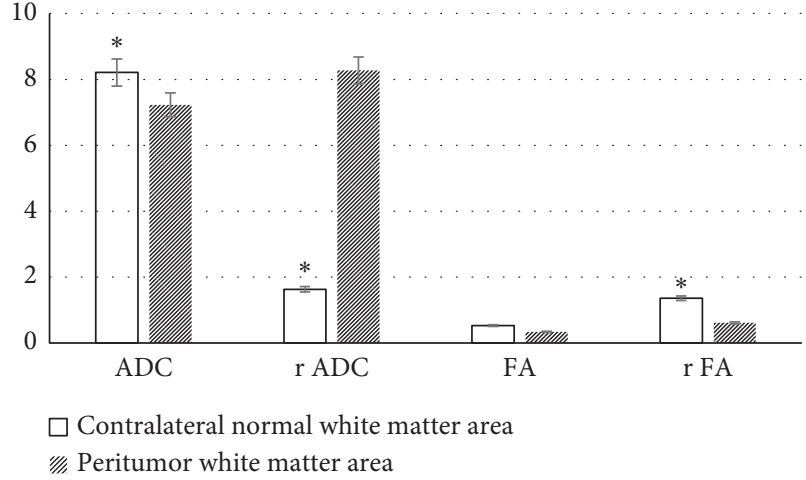

FIgURE 5: Comparison of various indicators of the contralateral normal white matter area and peritumoral white matter area. Note: ${ }^{*}$ indicated that the difference was obvious in contrast with the peritumor white matter area group $(P<0.05)$.

shown in Figure 6, the total cut rate of the experimental group was obviously greater than $\operatorname{Ctrl}(P<0.05)$.

3.6. Comparison of Postoperative Disability Rate. After surgical resection, the status of the postoperative limb motor function was observed. In experiment group, 30 cases showed normal postoperative limb motor function, and 2 cases had postoperative limb motor function decline, and the disability rate was $6.25 \%$. In Ctrl, 20 cases had normal postoperative limb motor function, and 11 cases had decreased limb motor function after operation, and the disability rate was $34.38 \%$. As shown in Figure 7, the disability rate of the experimental group was significantly lower than that of the Ctrl $(P<0.05)$.

\section{Discussion}

The cranium is the most complex organ in the human body, and it is also a site prone to cause tumors. Glioma, meningiomas, and metastases are common intracranial tumors, and different tumors have different treatment options. Choosing an effective treatment method requires accurate characterization of the tumor before surgery [14]. With the advancement of magnetic resonance technology, DTI is further developed on the basis of DWI. FA value, ADC value, r FA value, and $r$ ADC value are commonly used. Different types of tumors have different DTI parameters. Therefore, the tumor can be accurately characterized by measuring parameters $[15,16]$. Using segmentation technology to process the DTI map and extract a specific area in the image map can more accurately analyze the anatomical structure of the human brain [17]. The FCM algorithm has randomness in the selection method of the initial center point. Therefore, a combined method of maximum and minimum distance was proposed to determine the initial center point. Then, the FCM algorithm was optimized to improve the accuracy of tissue region segmentation in DTI data.

In this study, the optimized FCM algorithm was applied to DTI images of benign and malignant intracranial tumors. It was found that the image segmented by the traditional 


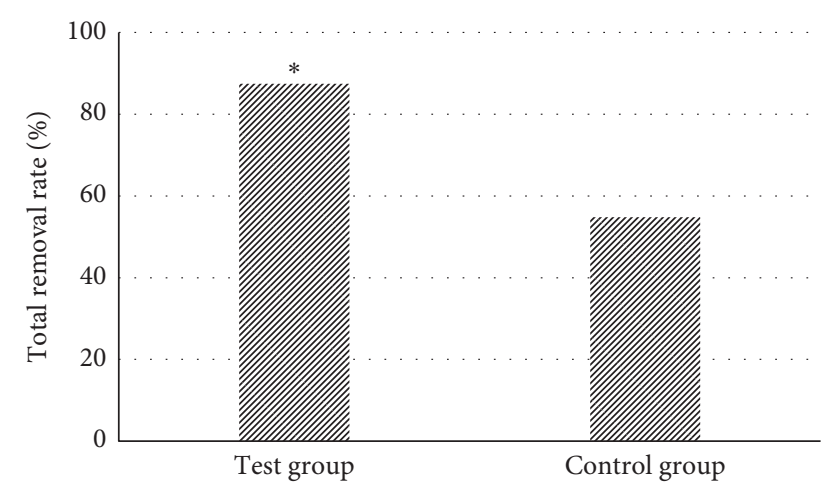

Figure 6: Comparison of total cut rate. Note: ${ }^{*}$ meant that the difference was obvious in contrast with the Ctrl $(P<0.05)$.

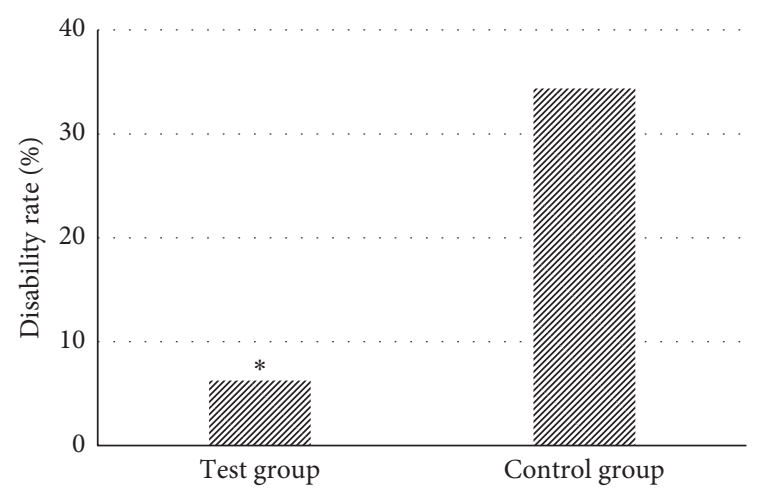

Figure 7: Comparison of the disability rate. Note. ${ }^{*}$ meant the difference was statistically significant compared with the control group $(P<0.05)$.

FCM algorithm did not have a clear contour and there were more areas with segmentation errors in contrast with the optimized FCM algorithm. It can be inferred that the optimized FCM algorithm had high accuracy. It is similar to the research results of Alexopoulos et al. (2019) [18]. The morphological pyramid was used to optimize the FCM algorithm, and the accuracy was also improved. In the study of 63 patients with intracranial space, there were 31 cases of glioma, 19 cases of meningioma, and 13 cases of metastases. There were obvious differences in ADC values, $\mathrm{r}$ ADC values, and $\mathrm{r}$ FA of meningioma, glioma, and metastases $(P<0.05)$. Besides, there were not obvious differences in the FA values of the three $(P>0.05)$. The ADC value, $\mathrm{r}$ ADC value, and $\mathrm{r}$ FA value of the benign tumors were larger than malignant tumors $(P<0.05)$, but there was no obvious difference between the FA values of benign tumors and malignant tumors $(P>0.05)$. The difference in ADC value and $\mathrm{r}$ FA value of the contralateral normal white matter area and peritumoral white matter area of the malignant tumor was obvious $(P<0.05)$, and there was no obvious difference in FA value of the two $(P>0.05)$. It was similar to the research results of Kumar et al. (2015) [19]. Therefore, it can be inferred that $r$ ADC value, ADC value, and $r$ FA value were useful in the diagnosis of benign and malignant tumors. After operation, the total resection rates of the experimental group and the Ctrl were $87.5 \%$ and $54.84 \%$; the disability rates were $6.25 \%$ and $34.38 \%$. There was an obvious difference between the total resection rate and the disability rate of the two groups $(P<0.05)$. It can be inferred that DTI based on the optimized FCM algorithm was accurate in the operation of patients with intracranial tumors and can remove the tumor to the greatest extent.

\section{Conclusion}

In the study, first, a method of combining the maximum and minimum distances was proposed, to improve the traditional FCM clustering algorithm for DTI tumor image segmentation. Then, the optimized FCM clustering algorithm was applied to the DTI imaging diagnosis of intracranial benign and malignant tumors. It was found that the optimized FCM algorithm improves the accuracy of the required area and ensures the stability of the segmentation results. In the DTI imaging diagnosis of intracranial benign and malignant tumors, the $\mathrm{r} A \mathrm{DC}$ value, $\mathrm{ADC}$ value, and $\mathrm{r}$ FA value of DTI are useful in the diagnosis of benign and malignant tumors, and DTI can improve the resection rate of tumors and the disability rate of patients, which is valuable in the operation of patients with intracranial tumors.

\section{Data Availability}

The data used to support the findings of this study are available from the corresponding author upon request.

\section{Conflicts of Interest}

The authors declare no conflicts of interest.

\section{References}

[1] M. Körner, M. Roos, V. S. Meier et al., "Radiation therapy for intracranial tumours in cats with neurological signs," Journal of Feline Medicine \& Surgery, vol. 21, no. 8, pp. 765-771, 2019.

[2] P. Jibrin, K. Ibebuike, and A. N. Ado-Wanka, "Histo-pathological pattern of intracranial tumours in the national hospital, Abuja," African Health Sciences, vol. 18, no. 2, pp. 281-286, 2018.

[3] D. Mata-Mbemba, J. Donnellan, P. Krishnan, M. Shroff, and P. Muthusami, "Imaging features of common pediatric intracranial tumours: a primer for the radiology trainee," $\mathrm{Ca}$ nadian Association of Radiologists Journal, vol. 69, no. 1, pp. 105-117, 2018.

[4] C. L. Mariani, T. A. Schubert, R. A. House et al., "Frameless stereotactic radiosurgery for the treatment of primary intracranial tumours in dogs," Veterinary and Comparative Oncology, vol. 13, no. 4, pp. 409-423, 2015.

[5] U. Ekpene, M. Ametefe, H. Akoto et al., "Comparison of unsupervised classification methods for brain tumor segmentation using multi-parametric MRI," NeuroImage: Clinical, vol. 12, pp. 753-764, 2016.

[6] Y. W. Cho, R. P. Allen, and C. J. Earley, "Efficacy of ferric carboxymaltose (FCM) $500 \mathrm{mg}$ dose for the treatment of restless legs syndrome," Sleep Medicine, vol. 42, pp. 7-12, 2018.

[7] X. Bai, Y. Zhang, H. Liu, and Y. Wang, "Intuitionistic centerfree FCM clustering for MR brain image segmentation," IEEE 
Journal of Biomedical and Health Informatics, vol. 23, no. 5, pp. 2039-2051, 2019.

[8] X. Bai, Y. Zhang, H. Liu, and Z. Chen, "Similarity measurebased possibilistic FCM with label information for brain MRI segmentation," IEEE Transactions on Cybernetics, vol. 49, no. 7, pp. 2618-2630, 2019.

[9] R. Dabbagh and S. Yousefi, "A hybrid decision-making approach based on FCM and MOORA for occupational health and safety risk analysis," Journal of Safety Research, vol. 71, pp. 111-123, 2019.

[10] H. Ali, M. Elmogy, E. El-Daydamony, and A. Atwan, "Multiresolution MRI brain image segmentation based on morphological pyramid and fuzzy C-mean clustering," Arabian Journal for Science and Engineering, vol. 40, no. 11, pp. 3173-3185, 2015.

[11] M. An, Q. Sun, J. Hu, Y. Tang, and Z. Zhu, "Coastline detection with gaofen-3 SAR images using an improved FCM method," Sensors (Basel), vol. 11, no. 6, p. 18, 2018.

[12] B. Duraisamy, J. V. Shanmugam, and J. Annamalai, "Alzheimer disease detection from structural MR images using FCM based weighted probabilistic neural network," Brain Imaging and Behavior, vol. 13, no. 1, pp. 87-110, 2019.

[13] K.-S. Chuang, H.-L. Tzeng, S. Chen, J. Wu, and T.-J. Chen, "Fuzzy c-means clustering with spatial information for image segmentation," Computerized Medical Imaging and Graphics, vol. 30 , no. 1, pp. 9-15, 2006.

[14] N. M. Hazzaa, L. Mancini, J. Thornton, and T. A. Yousry, "Somatotopic organization of corticospinal/corticobulbar motor tracts in controls and patients with tumours: a combined fMRI-DTI study," NeuroImage: Clinical, vol. 23, Article ID 101910, 2019.

[15] E. Aliotta, H. Nourzadeh, and P. P. Batchala, "Molecular subtype classification in lower-grade glioma with accelerated DTI," AJNR. American journal of neuroradiology, vol. 40, no. 9, pp. 1458-1463, 2019.

[16] C. Mekkaoui, P. Metellus, W. J. Kostis et al., "Diffusion tensor imaging in patients with glioblastoma multiforme using the supertoroidal model," PLoS One, vol. 11, no. 1, Article ID e0146693, 2016.

[17] K. S. Holly, J. S. Fitz-Gerald, B. J. Barker et al., "Differentiation of high-grade glioma and intracranial metastasis using volumetric diffusion tensor imaging tractography," World Neurosurgery, vol. 120, pp. e131-e141, 2018.

[18] G. Alexopoulos, U. Cikla, N. El Tecle et al., "The value of white matter tractography by diffusion tensor imaging in altering a neurosurgeon's operative plan," World Neurosurgery, vol. 132, pp. e305-e313, 2019.

[19] S. N. Kumar, A. L. Fred, and P. S. Varghese, "Suspicious lesion segmentation on brain, mammograms and breast MR images using new optimized spatial feature based super-pixel fuzzy C-means clustering," Journal of Digital Imaging, vol. 32, no. 2, pp. 322-335, 2019. 